\title{
Hand rejuvenation using standard Intense Pulsed Light (IPL) in Asian patients
}

\author{
Seiichi Maruyama, M. D.
}

Hills Aesthetic and Cosmetic Clinic

\begin{abstract}
Background: In recent years, facial rejuvenation using Intense Pulsed Light (IPL) is in demand and there have been many reports. However, hand rejuvenation, which aims to treat lentigines on the dorsal hand and wrinkles, is in demand but with few reports. In this study, hand rejuvenation was attempted using two wavelengths of a standard IPL system. It focuses mainly on improvement of lentigines, and efficacy on wrinkles on the dorsal hand will be discussed as well.

Subjects and Methods: The subjects were 128 Japanese patients (average age 58.1) who had lentigines and wrinkles on the dorsa of both hands, and the wavelength spectra of $560-1200 \mathrm{~nm}$ and $515-1200 \mathrm{~nm}$ were applied. In general, the number of treatments was not fixed, and further treatments beyond the first treatment were added only when asked for by the patient. Lentigines were assessed on 4 levels and wrinkles on 3 levels.

Results: The results in lentigines were graded "Excellent" in 45.3\%, "Good" in 14.8\%, "Unchanged" in $37.5 \%$ and "Bad" in $2.3 \%$ of the patients. Dark-toned lentigines in particular improved after one treatment. On the other hand, the results in wrinkles were graded as "Effective" in 25.0\%, "Hard to tell" in $39.1 \%$ and "Ineffective" in $35.9 \%$ of the patients after the first treatment, but there was increased improvement in some cases after multiple treatments.

Conclusions: By using appropriate IPL parameters with specific cut-off filters, dark-toned and flat senile lentigines could be treated effectively with a small number of treatments. On the other hand, improvement of fine dorsal wrinkles was noticed but more finesse is needed as to how to apply IPL and to decide on the number of treatments. Further study is necessary, but overall it can be said that IPL is an effective choice for hand rejuvenation.
\end{abstract}

Key words: Asian patients - dorsum of the hand - Intense Pulsed Light • senile lentigines • dorsal wrinkles

\section{Introduction}

In recent years, Japanese patients with lentigines and fine facial wrinkles have been looking for facial rejuvenation treatment using Intense Pulsed Light (IPL) because of the short downtime. IPL treatment is in demand and is widely known. However, hand rejuvenation, which aims to treat dorsal lentigines and wrinkles on the hand, is in demand but with few reports and is not widely known. Recently, Sasaya et al. reported on improvement of dorsal senile lentigines using IPL but not on wrinkles ${ }^{1)}$. To the best of my

\section{Addressee for Correspondence:}

Seiichi Maruyama MD,

Hills Aesthetic and Cosmetic Clinic

Taian Production Building, 7th Floor

21-8 Minami Fujisawa, Fujisawa City,

Kanagawa Prefecture 251-0055 Japan

TEL: (+81) 466-55-6737 FAX: (+81) 466-55-6757

E-mail: seiichi526@gmail.com knowledge, there have been no reports which have focused on improvement of wrinkles on the dorsal hand. Based on my experience in facial rejuvenation using IPL ${ }^{2)}$, I performed hand rejuvenation using IPL with two cut-off filters delivering specific polychromatic spectra. The study focuses on improvement of dorsal lentigines but also encompasses treatment of dorsal wrinkles. The method, results over time and conclusions are reported with a review of the relevant literature.

\section{Materials and Methods}

\section{Objective:}

The efficacy of hand rejuvenation in Japanese patients was assessed using 2 of the 7 cut-off filters available on

Received date: October 23rd, 2015

Accepted date: March 15th, 2016 
an IPL system (560 nm, $515 \mathrm{~nm})$. Irradiation was performed on the dorsum of the hand, targeting senile lentigines of different tones and sizes and various wrinkles.

\section{Overview of IPL:}

IPL was developed as a treatment device for telangiectasia applying the principle of selective photothermolysis, whereby the hemoglobin in the target blood vessels selectively absorbed the incident light at specific wavelengths, thereby damaging the blood vessel while sparing the surrounding skin. Goldman et al. introduced IPL first in America ${ }^{3}$ ), and the concept swiftly spread to Europe. Now there is a wide variety of IPL devices 4,5$)$. The light source of the IPL system is a xenon flashlamp. Unlike laser energy, the energy emitted by IPL systems is noncoherent, has spectral jitter and produces a spectrum of polychromatic light from shortwave visible (e.g., $515 \mathrm{~nm}$ ) to the near-infrared $(1200 \mathrm{~nm})^{6}$ ). Lasers emit at a single wavelength, so the appropriate system to use in clinical practice varies depending on the targets, namely pigmented lesions and vascular lesions. However, as already mentioned, IPL systems deliver polychromatic energy, therefore it has been reported that a wide variety of targets can be treated with a single IPL system, including pigmented lesions often referred to as "darkening of skin tone" or "uneven skin tone" (further referred to as diffuse melanin pigmentation) and senile lentigines, vascular legions, widening of pores, elastotic skin, rough skin, and fine wrinkles $2,6-9$ ). In addition, IPLs deliver lower fluences than lasers, so powerful photoablation and coagulation do not generally occur, and treatment with minimal downtime is possible ${ }^{2}$.

\section{Selective photothermolysis with IPL in pigment- ed lesions:}

In lesions where there are excessive deposits of melanin in the epidermis, the mechanism of pigment elimination differs between IPLs and Q-switched lasers. The Q-switched laser has an ultrashort pulse duration in the nanosecond range, which enables to heat up pigment in a target within the thermal relaxation time of the target, thereby selectively disrupting and destroying melanin-containing cells and structures resulting in regeneration of normal epidermis 10,11). IPL systems, on the other hand, emit pulse durations in the millisecond range, so the damage extends slightly beyond the target cell or structure, resulting in controlled coagulation under a microcrust, and the pigmented lesion is cleared through the natural turnover of the epidermis and shedding of the microcrust $6,12,13$ ).

\section{Collagenesis with IPL:}

There is a near-infrared component in the broadband spectrum delivered by an IPL system, so that segment of the spectrum is absorbed also by water too. Therefore, in the dermis, inflammation from a mild burn stimulates the fibroblast and facilitates collagen generation, thus skin-rejuvenation can be attained 14, 15). In fact, Bitter and Goldman reported an increase in collagen fiber through a histopathological study ${ }^{16)}$. Babilas, Negishi and Feng reported an increase in collagen using IPL by type I, type III collagen staining 4, 6, 17, 18). However, Negishi stated that, although increase in collagen skin texture and fine wrinkles improved, the effective area was vast so proper wavelengths and pulse duration remained uncertain ${ }^{19)}$. When I performed treatment on wrinkles around the eyelid using an IPL device (Vasculight ${ }^{\circledR}$, LUMENIS Corp.), I reported the efficacy of the $550-1200 \mathrm{~nm}$ wavelength spectrum. It was well received by patients, commenting such as "the wrinkles seem smaller" and "skin firmness is better and wrinkles are less noticeable 2)".

\section{Subjects:}

The subjects comprised 127 females and one male (total of 128) Japanese patients with lentigines on the dorsa of both hands who also wanted their wrinkles treated as well. The average age was $58.1 \mathrm{yrs}$ and all patients gave written informed consent to participate in this study on treatment effectiveness. Lentigines varied from brownish dark-toned to light-toned. Shapes were flat or protrusive, $1 \mathrm{~mm}$ to $10 \mathrm{~mm}$ in size [Figure 1left]. The size of the wrinkles varied, based on the ones that patients asked to be treated [Figure 1-right]. The study was performed in compliance with the Declaration of Helsinki.

\section{Treatment protocol:}

In general, the number of treatments was not fixed. Additional treatments beyond the first one were added only when the patient requested them, an essential component in this study to maintain fairness and objectivity. If there were two or more treatments, treatment was done at 1-month intervals. The follow-up period was set at least 6 months after the last treatment. Since the number of treatments was based on the patients' desires, the number of "patients registered for treatment," "patients who completed treatment, satisfied," and "patients who stopped treatment, unsatisfied" and the reasons why are reported as well. 


\section{ORIGINAL ARTICLES}

\section{IPL system:}

The Lumenis One ${ }^{\circledR}$ by LUMENIS Corp. (Israel) was used [Figure 2-left]. This system is equipped with 7 cut-off filters: $515 \mathrm{~nm}, 560 \mathrm{~nm}, 590 \mathrm{~nm}, 615 \mathrm{~nm}, 640$ $\mathrm{nm}, 695 \mathrm{~nm}$, and $755 \mathrm{~nm}$ [Figure 2-right-upper] Shorter wavelengths than the chosen filter are cut off, and longer wavelengths up to $1200 \mathrm{~nm}$ are within the emitted spectrum. However, higher intensities are seen in the shorter visible light waveband than in the nearinfrared, so the incident intensity is inconsistent across the spectrum ${ }^{6}$ ). For example, when the 560 nm cut-off filter is used, the light intensity peaks around and down to $560 \mathrm{~nm}$, and it is suitable for improving pigmented lesions because there is effective absorption by melanin at that wavelength [Figure 2-right-lower]. On
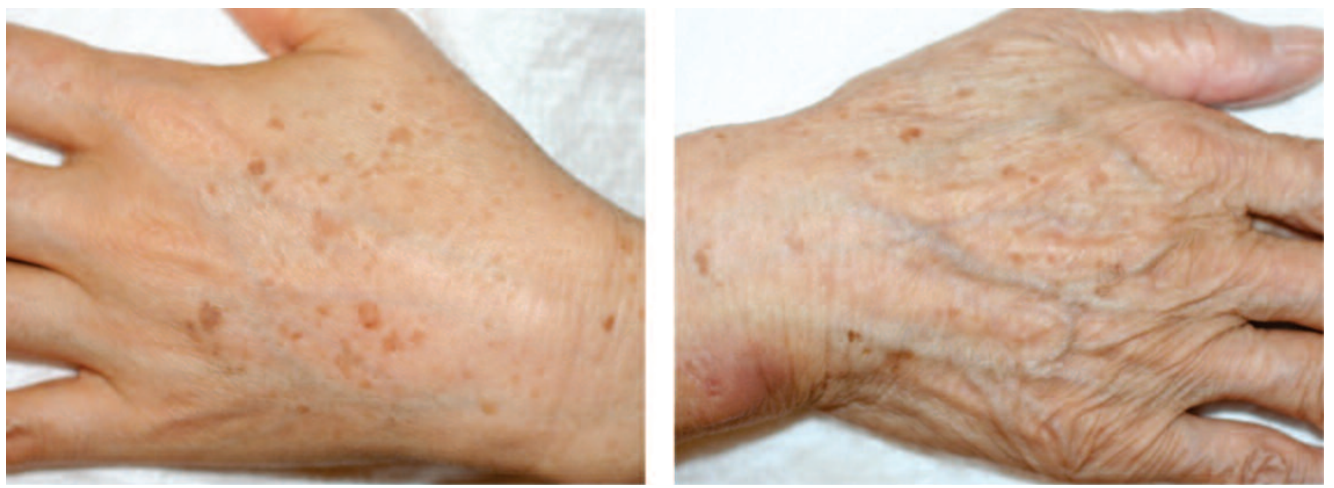

Fig. 1: The left and right panels show typical baseline findings of the dorsa of the left and right hands, respectively, in a 60-year-old woman (left) and a 75-year-old woman (right). Numbers of lentigines of various sizes and fine wrinkles can be seen.
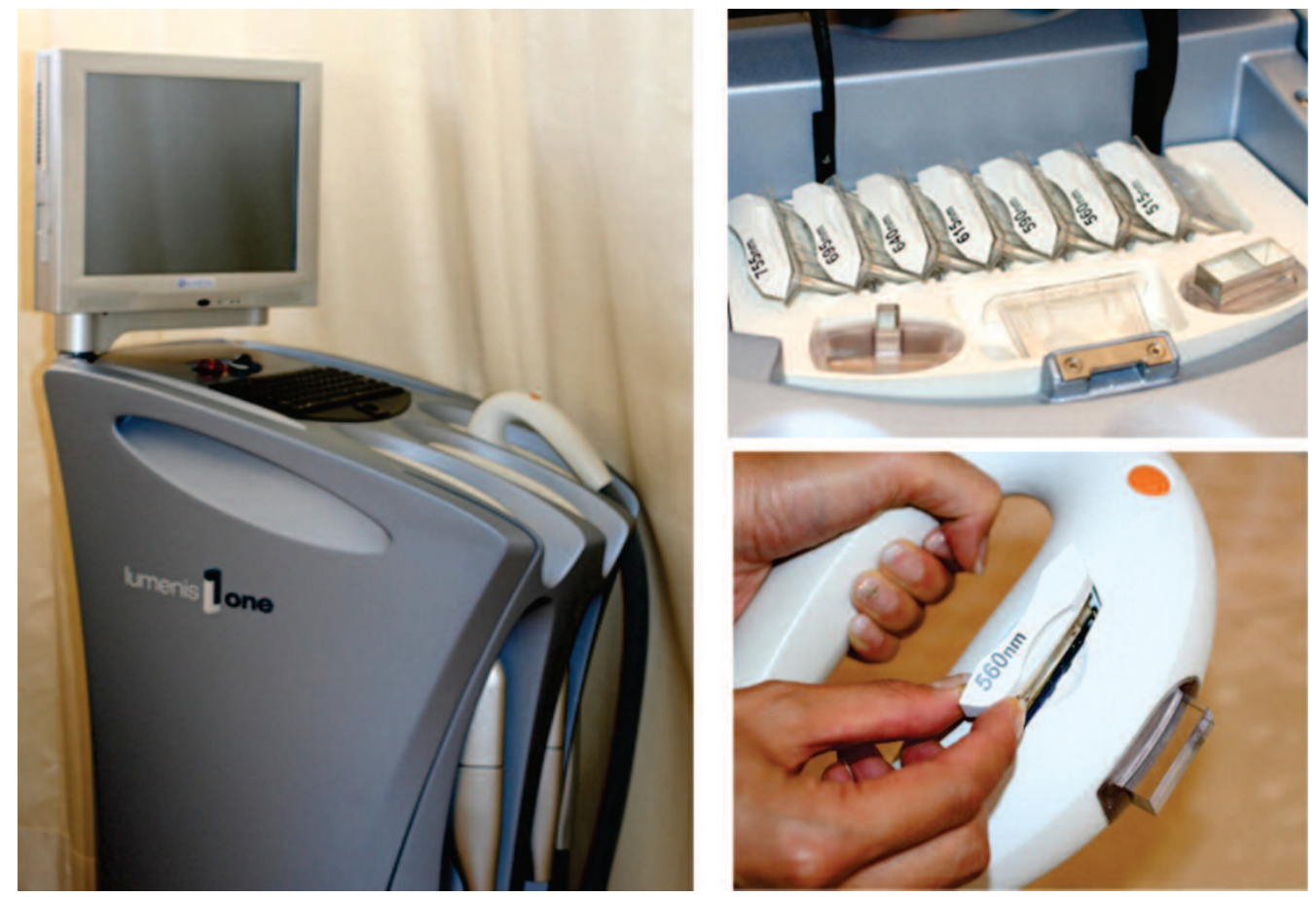

Fig. 2: [Left]: The standard IPL system used in the present study (Lumenis One ${ }^{\circledR}$, Lumenis, Israel). [Upper right]: The 7 cut-off filters supplied with this system: $515 \mathrm{~nm}$ (green), $560 \mathrm{~nm}$ (short-wave yellow), 590 $\mathrm{nm}$ (long-wave yellow), $615 \mathrm{~nm}$ (orange), $640 \mathrm{~nm}$ (red), $695 \mathrm{~nm}$ (ruby red), $755 \mathrm{~nm}$ (near-infrared). [Lower right]: The $560 \mathrm{~nm}$ cut-off filter being inserted into the IPL handpiece, to provide a polychromatic waveband of $560 \mathrm{~nm}-1200 \mathrm{~nm}$. 
the other hand, in the waveband up to $1200 \mathrm{~nm}$ the energy is also absorbed by both hemoglobin and water, so using this characteristic allows the physician to treat a range of symptoms such as vascular legions, skin elastosis, rough skin, and fine wrinkles $2,6-9)$. The system used in the present study obtained the Europe CE mark in March 2004 and FDA approval in July 2006. In Japan it is not approved by the Japanese Ministry of Health, Labour and Welfare, so physicians must individually import the device.

\section{Treatment and parameters:}

Before irradiation, patients are told to wash their hands with soap and water to remove any sunscreen or hand cream. A cooled coupling gel is then spread over the entire dorsum. The $560 \mathrm{~nm}$ and $515 \mathrm{~nm}$ cut-off filters are selected.

\section{$560 \mathrm{~nm}:$}

The $560 \mathrm{~nm}$ filter was chosen first to improve not only senile lentigines and diffuse melanin pigmentation but also wrinkles [Figure 3-left]. The parameters were: emitted spectrum, $560-1200 \mathrm{~nm}$; double pulse mode; pulse duration $4.0 \mathrm{~ms}$ each pulse with a delay of 30 $\mathrm{ms}$ ); spot size $8 \times 35 \mathrm{~mm}$; and fluence $12 \mathrm{~J} / \mathrm{cm}^{2}$. A single shot was delivered to each target area, and the handpiece moved on to eliminate overlap. The clinical end point was slight erythema of the entire dorsum.

\section{$515 \mathrm{~nm}$ :}

The filter was then changed to the $515 \mathrm{~nm}$ cut-off, at which wavelength there is better absorption by melanin. The parameters were: emission spectrum, 515 - $1200 \mathrm{~nm}$; single pulse mode, pulse duration $3.0 \mathrm{~ms}$; spot size $8 \times 15 \mathrm{~mm}$; and fluence $13 \mathrm{~J} / \mathrm{cm}^{2}$. Only the lentigines were targeted with a single shot, or two shots if required. The end point was a dark change in the lentigines. In order to limit the incident energy to the target lentigines and spare the normal skin as much as possible, I used a piece of white card as a mask, with small holes cut out to match the sizes of the lentigines [Figure 3-right]

\section{Post-treatment care:}

Immediately after irradiation, the back of the hand was cooled with a cold towel and the application of gel for 30 minutes. After that, no gauze was used. Only hypoallergenic hand cream was allowed and the patients were told to wash their hands gently. If itching or erythema occurred, a mild steroid ointment was applied. Also, to limit observation of the efficacy of the IPL intervention, other bleaching agents such as hydroquinone and retinoic acid were not administered. Patients were advised to protect the treated area from exposure to sunlight with application of a UVA/B sunscreen with an SPF $>40$.

\section{Assessment}

The efficacy of the treatment was assessed by both the patients and myself, based on the photos taken with a digital camera under as much as possible the same conditions. In general, photos were taken before treat-
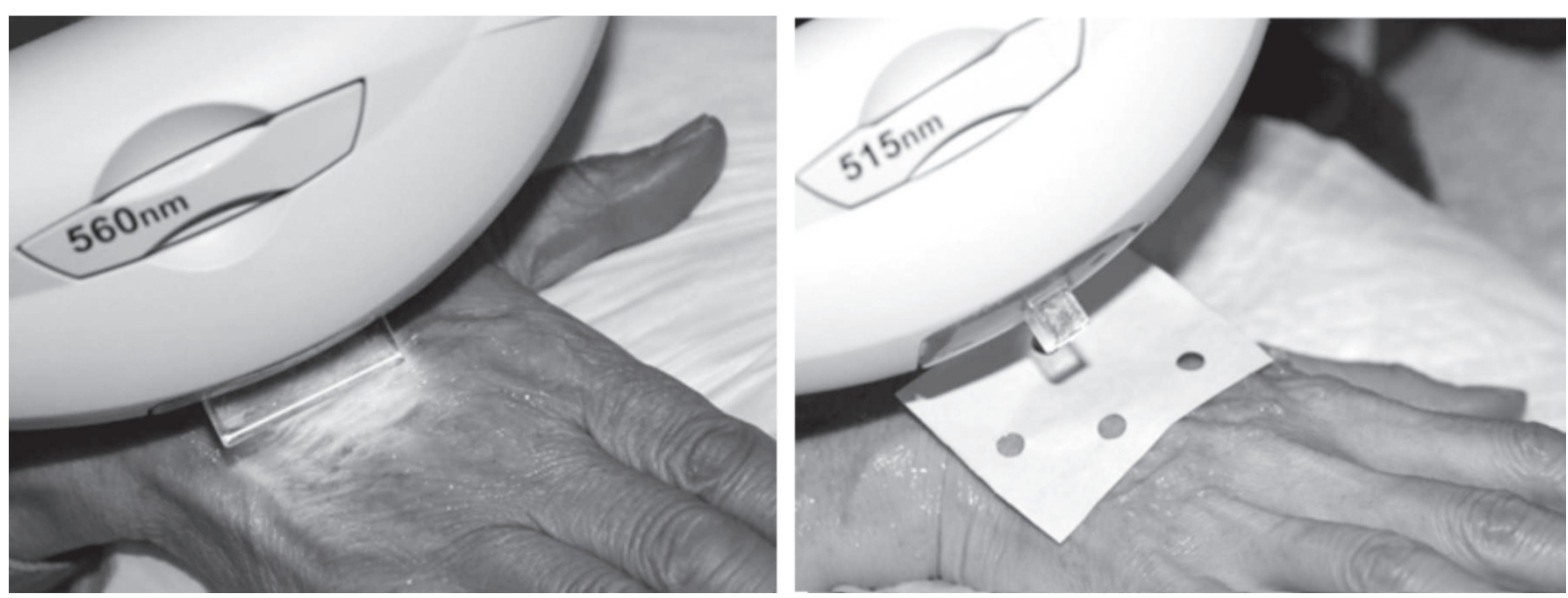

Fig 3: [Left]: IPL irradiation of the entire tissue target on the dorsum of the hand with the 560 cut-off filter.

[Right]: Use of the cut-out mask to limit irradiation to only the lentigo being targeted, thereby protecting the epidermal melanin in the surrounding normal tissue from exposure. 
ment, 14 days after treatment, and one month after treatment. If the patient continued treatment, this practice was also followed. In addition, a picture was taken at least 6 months after the last treatment, however, additional photos were taken when needed, such as premature desquamation of the microcrust.

Dorsal senile lentigines: Based on the photos, the results were evaluated in two ways: (1) checking each photo and the assessing the results according to the number of treatments; (2) overall judgment by comparing the photos at baseline and at least 6 months after the final treatment. The latter was assessed on 4 levels ("Excellent", improvement of lentigines after one treatment; "Good", improvement after two or more treatments; "Unchanged", no improvement regardless of the number of treatments; "Bad", darkening of the lentigines regardless of the number of treatments [Table.1A]).

Dorsal wrinkles: Based on the photos, the results were evaluated as for the lentigines. The overall improvement was assessed on 3 levels ("Effective", improvement noted by both patient and physician; "Hard to tell", either the physician or patient noted improvement, and "Ineffective", neither the physician nor the patient noted any improvement [Table.1-B]).

\section{Downtime:}

In general, downtime can be defined as; "the period of time where patients may choose not to appear in public due to expected side effects such as erythema ${ }^{20)}$." Many patients said they could not show their hands in public until the microcrusts had shed. Therefore, in this particular study I defined downtime as; "the period until all microcrusts on the dorsal hand shed completely." Downtime was assessed only after the first treatment, based on the photo taken 14 days after treatment. "Good", less than 14 days; and "Poor", 14 days or more [Table.1-C]

\section{Post-inflammatory hyperpigmentation (PIH):}

Post-inflammatory hyperpigmentation was assessed only after the first treatment, based on the photos. The erythema and pigmentation were compared at the time of microcrust desquamation and one month after irradiation. "Yes", an increase in PIH was noted; "No", no increase was noted [Table.1-D]

\section{Results}

The average number of treatments was 1.51, and the average treatment period was 9.81 months.

Table 1: Assessment criteria for lentigines, wrinkles, downtime period and presence/absence of postinflammatory hyperpigmentation.

\begin{tabular}{lll}
\hline Item & Criteria & Assessment \\
\hline \multirow{2}{*}{ Lentigines } & Number of lentigines less and/or lighter tone post 1 Tx & Excellent \\
\cline { 2 - 3 } & No change in either number or tone of lentigines regardless of Tx No. & Good \\
\cline { 2 - 3 } & Darkening of lesions regardless of Tx No & Bad \\
\hline \multirow{2}{*}{ Wrinkles } & Either patient or clinician notes improvement & Effective \\
\cline { 2 - 3 } & Neither patient nor clinician notes improvement & Hard to tell \\
\hline Downtime & Less than 14 days & Ineffective \\
\cline { 2 - 3 } & 14 or more days & Good \\
\hline PIH ${ }^{*}$ & Increase in erythema or pigmentation & Poor \\
\cline { 2 - 3 } & No increase in erythema or pigmentation & Yes \\
\hline
\end{tabular}

*Presence of pigmentation or erythema assessed at 1 month post-Tx

Note: Patients having more than $1 \mathrm{Tx}$ had an inter-Tx period of 1 month. Final assessment was performed at least 6 months post-final Tx 


\section{Dorsal senile lentigines:}

Based on the number of treatments, 73 cases (57.0\%) showed improvement after one or two treatments. Dark-toned flat lentigines cleared particularly well. Forty-eight patients (excluding the 3 out of 51 cases in whom the result was "Bad") did not continue treatment because "further improvement of the tone cannot be expected" [Table 2]. Light-toned and protrusive lentigines did not show improvement. The overall assessment was as follows: "Excellent", 45.3\%; "Good", 14.8\%; "Unchanged", 37.5\%; and "Bad" 2.3\% [Figure 4-A]. Although not included in the evaluation, 28 out of the 48 patients $(58.3 \%)$ who were in the "Unchanged" group commented that their overall skin tone was better.

\section{Dorsal wrinkles:}

Based on the number of treatments, the assessment by patient and physician differed dramatically after the first treatment. Whereas 40 patients noted subjective improvement, only 2 cases improved in the physician's eyes [Table 2]. The former patients answered, "My skin is more resilient," "My skin tone is better and the wrinkles seem smaller," "My skin was smoother when I applied hand cream" and so on. The cases that resulted in improvement by both patient and physician required two or more treatments. Fine wrinkles improved, deep wrinkles did not. In two cases, after 5 or 6 treatments the patients were satisfied that the wrinkles had improved [Table.2]. The overall assessment was as follows: "Effective", 25.0\%; "Hard to tell", 39.1\%; and "Ineffective", 35.9\% [Figure 4-B]

\section{Downtime:}

The downtime assessment was "Good" in 20.3\% and "Poor" in $79.7 \%$ [Figure 4-C]. There were cases where it took more than 20 days for the microcrust to shed completely. In the case of dark-toned lentigines in particular, the downtime was longer.

\section{PIH:}

Twenty-five percent of the patients answered "Yes", but $75 \%$ answered "No". It was a fine result [Figure 3D]. Interestingly, all those who had PIH all experienced partial microcrust desquamation within 6 days

\section{[Figure 5-left, right; ].}

Downtime and post-inflammatory hyperpigmentation were assessed only after the first treatment. However, if the lentigines improved after two or more treatments it was less likely that microcrusts formed. Even when they did, downtime was less than 14 days and PIH occurred less.

\section{Complications:}

Complications were assessed after the first treatment. Most common was erythema in 34 cases (26.6\%), followed by post-inflammatory hyperpigmentation in 32 cases (25.0\%). There was also itching in 11 cases (8.59\%), blister formation in 3 cases $(1.56 \%)$ and scarring in 1 case $(0.78 \%)$ [Table 3 ]. The scar was probably due to incomplete cooling after irradiation, or partial desquamation of the microcrust one day after irradiation but the patient did not report it to the clinic and left the area without proper care until consultation 2 weeks later. No scarring was seen in cases with two or

Table 2: Patient and clinician satisfaction based on the number of treatments

\begin{tabular}{|c|c|c|c|c|c|c|c|}
\hline \multirow{3}{*}{ No of $T x$} & \multirow{3}{*}{$\begin{array}{c}\text { No of Patients } \\
\text { (Completed } \\
\text { Tx })\end{array}$} & \multicolumn{5}{|c|}{ Improvement } & \multirow{3}{*}{$\begin{array}{l}\text { Unsatisfied } \\
\text { patients* }\end{array}$} \\
\hline & & \multirow{2}{*}{ Lentigines } & \multicolumn{4}{|c|}{ Wrinkles, assessed by: } & \\
\hline & & & Patient \& Clinician & Patient & Clinician & Neither & \\
\hline 1 & $128(84)$ & 58 & 2 & 40 & 2 & 40 & 26 \\
\hline 2 & $44(31)$ & 15 & 19 & 27 & 21 & 4 & 16 \\
\hline 3 & $13(8)$ & 3 & 7 & 7 & 8 & 1 & 5 \\
\hline 4 & $5(3)$ & 1 & 2 & 2 & 3 & 1 & 2 \\
\hline 5 & $2(1)$ & 0 & 1 & 1 & 1 & 0 & 1 \\
\hline 6 & $1(1)$ & 0 & 1 & 1 & 1 & 0 & 1 \\
\hline Total & ---- & 77 & 32 & 78 & 36 & 46 & 51 \\
\hline
\end{tabular}

* These patients all stopped treatment because further improvement of lentigines tone was unlikely. All other patients completed treatment, and were to some degree satisfied. 


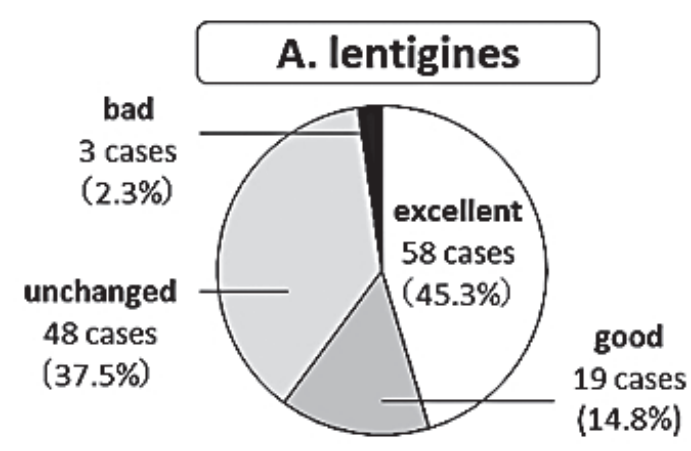

C. downtime period

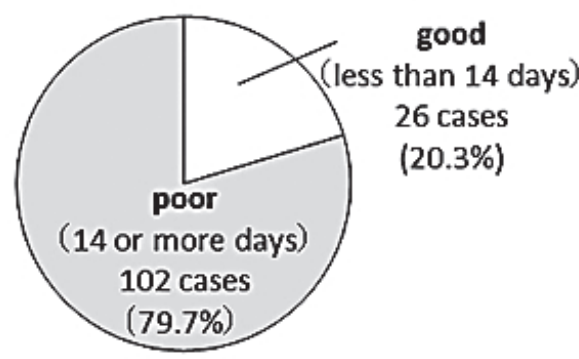

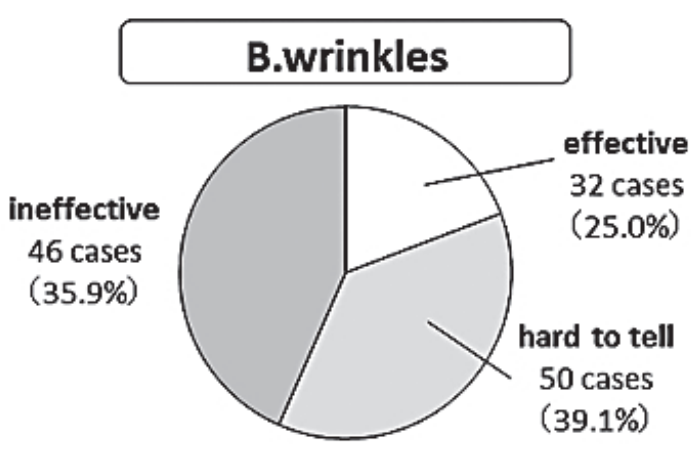

\section{D. post-inflammatory hyperpigmentation(PIH)}

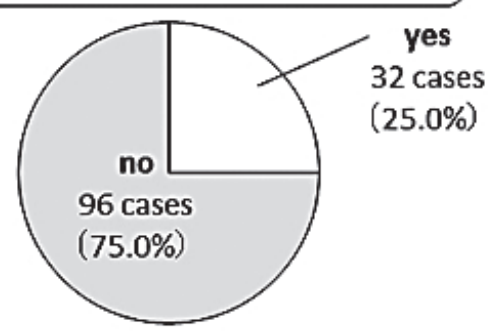

Fig. 4: Results of the comprehensive assessment. [A]: Lentigines. [B]: Wrinkles. [C]: Downtime. [D]: PIH
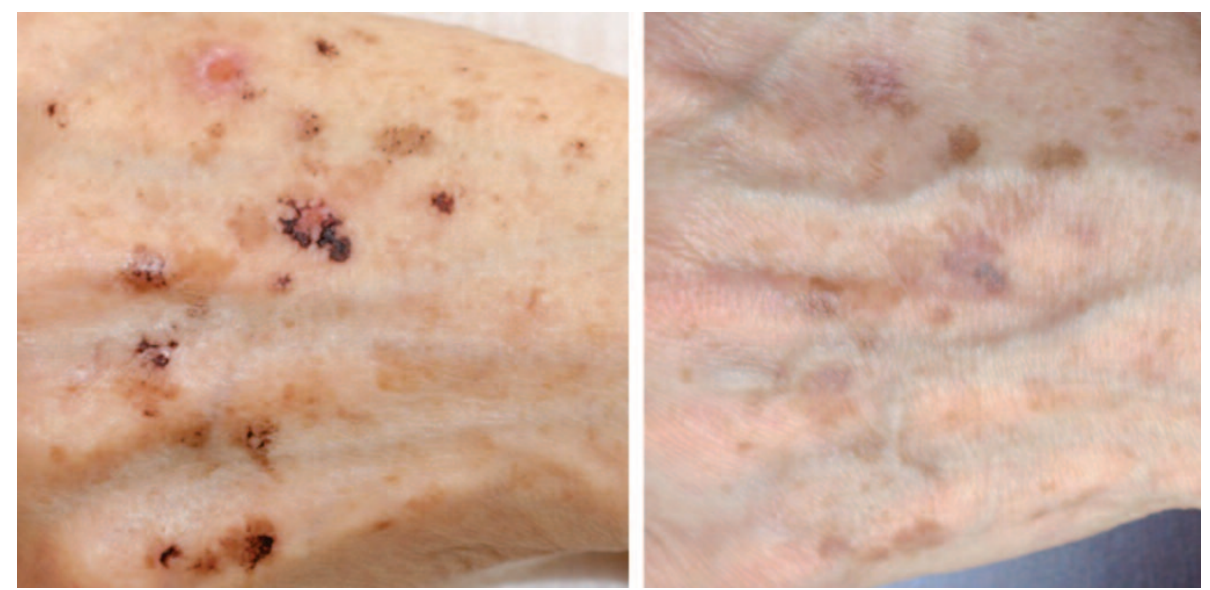

Fig. 5: [Left]: Findings on the dorsum of the left hand in a 65-year-old woman 6 days post-irradiation. Crusting is seen with partial exfoliation, regarded by the author as a less than optimum finding. The longer the crusting remains before falling off spontaneously, the less the chance of PIH as the crust acts as a biological dressing.

[Right] Same hand 1.5 months after irradiation. Inflammatory-related pigmentation is seen (PIH).

Table 3: Side effects

\begin{tabular}{ll}
\hline Side effect & No of Pats \\
\hline Itching & $11(8.59 \%)$ \\
\hline Erythema1 & $34(26.6 \%)$ \\
\hline PIH2 & $32(25 \%)$ \\
\hline Blister formation & $3(1.56 \%)$ \\
\hline Scar formation3 & $1(0.78 \%)$ \\
\hline
\end{tabular}

1: Erythema was noted following desquamification of the microcrust within 10 days post-Tx

2: Postinflammatory hyperpigmentation. PIH was noted following desquamification of the microcrust within 6 days post-Tx

3: Scarring was noted following desquamification of the microcrust one day after treatment 


\section{ORIGINAL ARTICLES}

more treatments, and complications such as erythema and itching occurred less.

\section{Representative cases}

\section{Case 1: 69-year-old woman [Figure 6-left].}

Treatment: one treatment on both dorsa. At 10 months after treatment, lentigines improved dramatically and did not reappear. Wrinkles showed no improvement by both patient and physician assessment [Figure 6right].

Assessment: lentigines "Excellent," wrinkles "Ineffective," downtime "Poor," and PIH "No."

\section{Case 2: 65-year-old woman [Figure 7-left].}

Treatment: two treatments on both dorsa. At 6 months after the second irradiation, partial clearance of the lentigines had been achieved with some improvement. Physician noted no improvement of wrinkles, but the patient remarked that her skin tone was better and wrinkles were less noticeable" [Figure 7-right] Assessment: lentigines "Good," wrinkles "Hard to tell," downtime "Poor," and PIH "No."

\section{Case 3: 68-year-old woman [Figure 8-left].}

Treatment: two treatments on both dorsa. At 12 months after the second irradiation, no improvement was seen
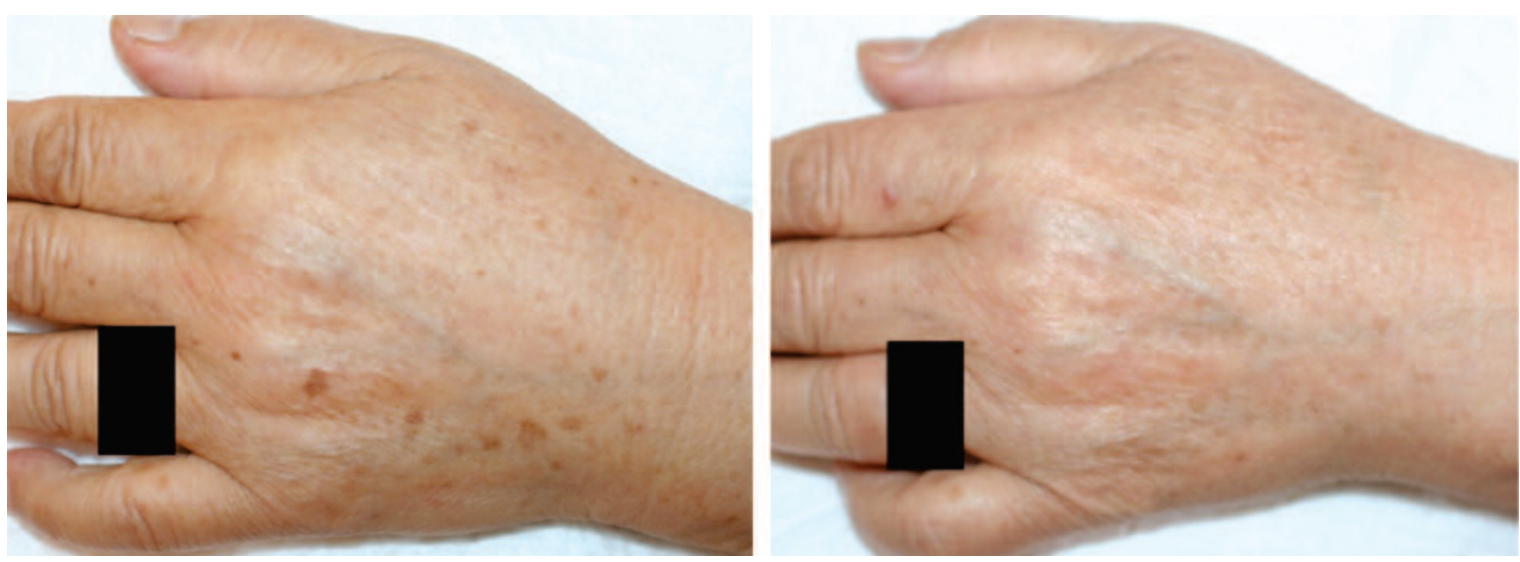

Fig. 6: [Left]: Baseline findings of the left dorsum in a 69-year-old woman. Numbers of lentigines of various sizes and wrinkles are seen.

[Right]: Same hand, 10 months post-treatment (single session).
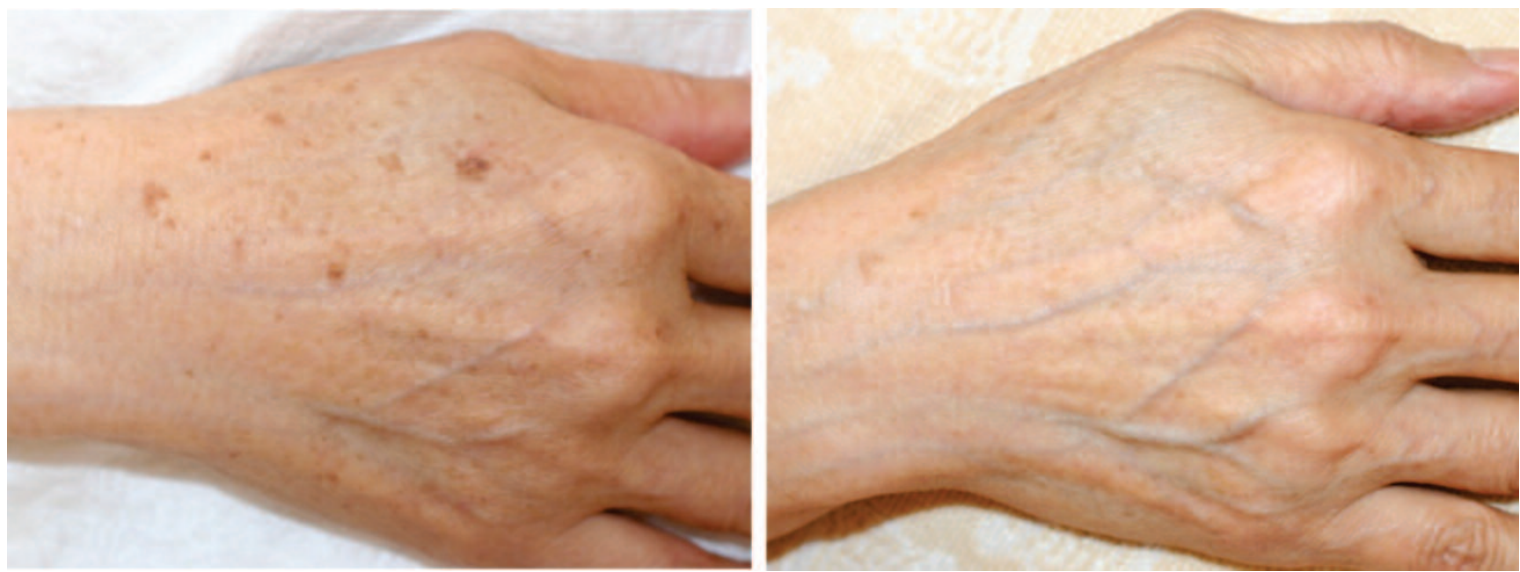

Fig. 7: [Left]: Baseline findings of the right dorsum in a 65-year-old woman. Numbers of lentigines of various sizes and wrinkles are seen.

[Right]: Same hand, 6 months after 2 treatments. Improvement in the lentigines is seen, but no improvement in the wrinkles. 
in the lentigines, but improvement of the wrinkles was assessed as "Effective" by both the patient and physician [Figure 8-right].

Assessment: lentigines "Unchanged," wrinkles "Effective," downtime "Poor," and PIH "No."

\section{Discussion}

Unlike Caucasians, many Asians have Fitzpatrick Type III-IV skin and tend to pose unique challenges when it comes to pigmentary disorders such as freckles, lentigines, melasma, pigmentation from chronic photoaging and so on. Irregularity in skin tone is a great cosmetic concern for Asian patients ${ }^{12)}$. Also, Tanaka et al. reported that prevention and management of postinflammatory hyperpigmentation (PIH) is of great inter- est in Asian skin, which has a tendency to form PIH due to the susceptibility of melanin overproduction as a protective measure by melanocytes following any inflammatory damage to the stratum basale ${ }^{21)}$. It has been reported that the Q-switched laser is quite effective in treating senile lentigines in Japanese patients, but care should be taken to prevent PIH after treatment ${ }^{22}$ ). A recent report suggests 2 or 3 months of pre-treatment, administering vitamin $\mathrm{C}$ and hydroquinone. This is said to lower the risk of post-inflammatory hyperpigmentation down to approximately 30\% even with output powers that cause the Immediate Whitening Phenomenon (IWP) ${ }^{22}$ ). Another report advises that we should use the laser at that output power and then administer hydroquinone and tretinoin as post-treatment care, and suggests that this approach
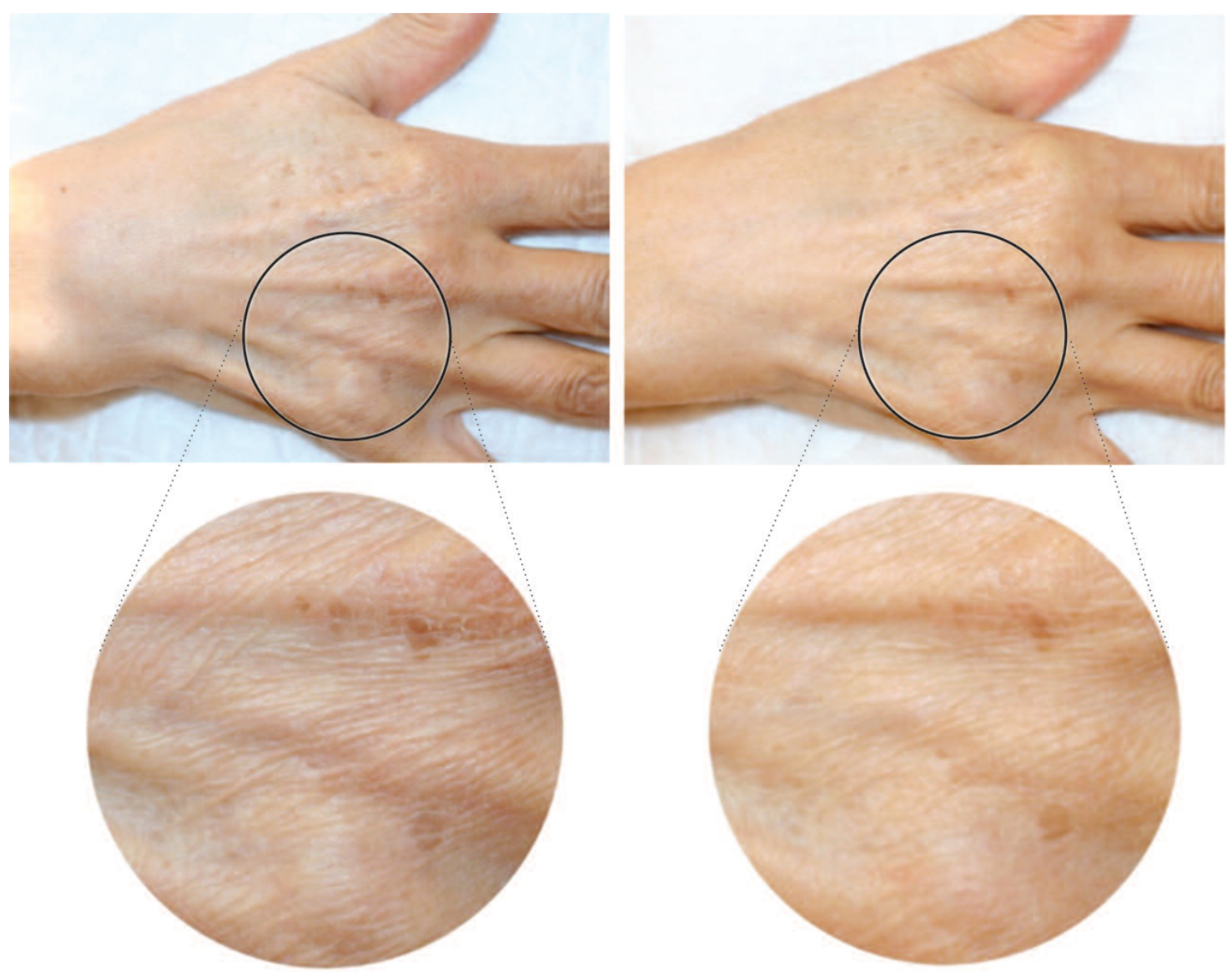

Fig. 8: [Left, upper and lower]: Baseline findings of the right dorsum in a 68-year-old woman. Numbers of lentigines of various sizes and wrinkles are seen. Note that none of the lentigines is particularly dark in color. A magnified view of the circled area is shown below.

[Right, upper and lower]: The same hand, 12 months after the last treatment (2 sessions). There is no improvement in the lentigines, but the wrinkles have been markedly improved as can be clearly seen in the magnified sector. 
will lower the risk of PIH to approximately 20\% 23) Both papers report that pre- or post-treatment will lower the PIH risk. However, the typical Japanese skin is such that, once post-inflammatory hyperpigmentation has occurred, it can persist for a long time, so caution is needed ${ }^{24)}$. Also, according to Sasaya et al., experience tells us that PIH occurs more on the dorsum of the hand than on the facial area after Qswitched laser treatment ${ }^{1)}$. That is probably why Qswitched laser treatment is not popular when treating senile lentigines on the dorsum. Lately, the effectiveness of IPL for that treatment has been reported, because of less invasiveness and shorter downtime than laser treatment. Only the wavelength of $515 \mathrm{~nm}$ was used and lentigines were treated multiple times at 1-month intervals 1,25 ).

Before this study, I have performed IPL treatment extensively on Japanese patients, to improve senile lentigines and wrinkles on the dorsum of the hand, using only the $560-1200 \mathrm{~nm}$ waveband and fixing the number of treatments from 3 to 5 . It was received relatively well by patients, as they would say; "My skin is more resilient," "The fine wrinkles are less noticeable," "My skin tone is better" and so forth. However, diffuse melanin pigmentation would improve but large lentigines did not improve as much, despite the multiple treatments. Also, there was some dissatisfaction from patients during the time of microcrust formation, saying "I cannot show my hands in public," and "A smaller number of treatments is preferable."

Therefore, I focused on improving dorsal senile lentigines and also sought to treat wrinkles by choosing two wavebands: $560-1200 \mathrm{~nm}$ and $515-1200$ $\mathrm{nm}$. The first choice, $560 \mathrm{~nm}$, is effectively absorbed by melanin and is suitable for treating pigmented lesions. In addition, although the cut-off filter ensures that the highest photon intensity is in the shorter yellow wavelengths, the waveband includes all wavelengths up to $1200 \mathrm{~nm}$ : water therefore also becomes a chromophore, thereby offering improvement of fine wrinkles and texture of the skin through collagenesis and dermal remodelling ${ }^{6}$ ). In my previous report, Facial Rejuvenation using Intense Pulsed Light (IPL), I used a different IPL system from the present study with a slightly shorter wavelength of $550 \mathrm{~nm}$, and performed 5 or 6 treatments to improve diffuse facial pigmentation and wrinkles around the eyelids. It was well received ${ }^{2)}$, so I chose $560 \mathrm{~nm}$ with the expectation that it would be effective in treating diffuse melanin pigmentation on the dorsum of hand or for specific lentigines, and somewhat effective in improving wrinkles. The other choice, $515 \mathrm{~nm}$, is a shorter wavelength than $560 \mathrm{~nm}$ so it is absorbed by melanin even more strongly. Therefore, when specific lentigines are targeted with high fluence, improvement is likely with a small number of treatments. In conclusion, taking advantage of the optimum optical characteristics of each wavelength allows the physician to perform hand rejuvenation of higher quality.

Results showed improvement of dorsal lentigines in 73 cases $(57.0 \%)$ within two treatments, so improvement is possible with a small number of treatments. Adding the "Excellent" and "Good" scores 77 cases (61.1\%) had satisfactory results, and in other cases patients would give positive comments such as; "My skin tone is better." Therefore, it can be said IPL is effective and can be recommended. However, patients should be informed beforehand that light-toned or protrusive lentigines are less likely to improve after only one or two treatments, and there is a possibility that they still may not improve after multiple treatments.

On the other hand, improvement of wrinkles on the dorsal hand was "Effective" in only 32 cases (25.0\%) and "Hard to tell" in 50 cases (39.1\%), so IPL at the parameters used in the present study is not necessarily effective. Patient satisfaction after one treatment was surprisingly high, many of them saying "My skin tone is better and wrinkles appear to be smaller." This is probably due to the clearance of diffuse melanin pigmentation and lentigines, and patients felt that the wrinkles improved too, because the hand looked better. Unlike lentigines, most cases where both the patient and physician noted improvement required two or more treatments. The improvement was in fine wrinkles. Fine wrinkles, like texture (structured ridge pattern of sulcus cutis and area cutanea), are defined as "depressions that do not reach the reticular dermis but only to the subpapillary dermis 26)." Mild photocoagulation from the IPL causes inflammation and stimulates the fibroblasts and thus collagen is generated ${ }^{6,14}$ 18). This likely improved the texture of the skin and fine wrinkles, further improved by the longer-term remodelling process.

As Kawada et al. have reported, microcrust desquamation very rarely occurs spontaneously in around the 10-day mark using this method ${ }^{27)}$. In fact, 102 cases $(79.7 \%)$ needed 14 or more days of downtime. Negishi reports that microcrust shedding takes about 14 days when treating the dorsal hand, almost twice the time needed in facial treatment ${ }^{28)}$. These results are matched in the present report, and suggest that, after IPL irradiation of the dorsum, epidermis turnover takes more time than the face and it delays microcrust desquamation, resulting in longer down- 
time. In this study, downtime is defined as "the period until all microcrusts shed completely," but some took 7 days and others 14 or more days, so downtime is not necessarily long. However, if the microcrust sheds early there is more risk of complications such as erythema and post-inflammatory hyperpigmentation so one needs to be careful. Considering how the long downtime affects the patient's daily life, it is important to explain beforehand to avoid trouble. In this study, all of the patients were fully informed in detail about the situation and care required after treatment, so a downtime of 14 or more days did not become an issue at all.

Post-inflammatory hyperpigmentation after IPL treatment occurred in 32 cases (25.0\%). Taking into consideration the fact that no additional pre- or posttreatment was performed, IPL offers a lower risk of PIH than Q-switched laser treatment. Also, if the microcrust does not desquamate early after treatment, PIH is unlikely to occur. Desquamation takes 14 days on the dorsal hand and 7 days on the face ${ }^{28)}$. IPL irradiation is performed in the millisecond range so the pigment within the cells itself is not a target even with relatively high output power, and the IWP does not occur. Therefore, it is considered that risks of complication such as PIH are low despite the long downtime. So, advising the patient to treat the dorsal hand gently is important to prevent premature microcrust desquamation, and the lack of PIH in $75 \%$ of the patients bears this out.

In the present study, the patients decided on the number of treatments by themselves, and some did not continue with the treatment because of unsatisfactory results on the improvement of lentigines. However, from a clinical standpoint, it can be said that an average of 1.5 treatments at the parameters used in the present study could deliver successful hand rejuvenation and would be good for the patient. Significance is to be noted especially in the treatment of lentigines, as the goal of "improvement with a small number of treatments" was achieved. As for wrinkles, patients were relatively satisfied after one treatment, but further IPL treatment is preferable to gain better results. Therefore the physician needs to work on giving patients as little downtime as possible and not let it affect their daily lives. First, we should focus on the treatment of pigmented lesions and improve them to some degree so that treatment afterwards is likely to have a shorter downtime. Then, it is easier to gain trust from the patients even if multiple treatments follow to further improve wrinkles. In addition, if improvement of pigmented lesions is attained, it is possible to use longer wavelengths than the $560 \mathrm{~nm}$ and $515 \mathrm{~nm}$ and the physician can treat wrinkles from a variety of choices with more effectiveness.

A high level of physician technique is necessary when using the IPL, since the irradiation settings are more varied than laser. It is said that the most appropriate wavelength and pulse duration remain uncertain 19), but this study shows that by using the characteristics of the two wavelengths, $515 \mathrm{~nm}$ and $560 \mathrm{~nm}$, IPL is an effective choice for hand rejuvenation in Asian patients.

\section{Conclusions}

In hand rejuvenation, through the use of carefully selected IPL cut-off filters and resultant wavebands, it is possible to improve dark-toned and flat senile lentigines with a small number of treatments. Fine dorsal wrinkles improved as well, but more study needs to be done in selecting the most appropriate IPL parameters and number of treatments. Further development is eagerly anticipated.

\section{Conflict of Interest}

None.

\section{References}

1: Sasaya H, and Kawada A: How to treat solar lentigines on the dorsal hand using IPL. In: (Kawada A, ed.) Laser Therapy for Skin Diseases. 2011, ChugaiIgakusha, Tokyo. pp 200.

2: Maruyama S, Ueda K, Mikura A, and Tsubakihara R (2003): Facial Rejuvenation using Intense Pulsed Light (IPL). Journal of Japan Society of Plastic and Reconstructive Surgery, 23, 4:232 - 239.

3: Goldman MP, and Eckhouse S (1996):

Photothermal Sclerosis of Leg Veins. Dermatologic Surgery, 22:323 - 330.

4: Babilas P, Schreml S, Szeimies RM, and Landthaler M (2010): Intense Pulsed Light (IPL): A Review. Lasers in Surgery and Medicine, 42:93 - 104.

5: Ash C, Town G, and Bjerring P (2008): Relevance of the Structure of Time-Resolved Spectral Output to Light-Tissue Interaction Using Intense Pulsed Light (IPL). Lasers in Surgery and Medicine, 40:83 - 92. 
6: Negishi K, and Matsunaga K (2010): Intense Pulsed Light Therapy in Aesthetic Dermatology. The Journal of Japan Society for Laser Surgery and Medicine, 31, 1:53 - 60.

7: Maruyama S, Ueda K, Oba S, and Omiya Y (2002): Treatment of facial Port-Wine Stain with Intense Pulsed Light (IPL). Journal of the Japan Society of Cranio-Maxillo-Facial Surgery, 18, 3:224 - 231.

8: Maruyama S, Ueda K, Oba S, Omiya Y, and Naka M (2002): Intense Pulsed Light (IPL) Treatment for Photo-Aging and Vascular Disease. The Journal of Osaka Medical College, 61, 2:27 - 35.

9: Maruyama S, Ueda K, Oba S, Omiya Y, and Naka M (2002): Treatment of Facial InflammatoryPigmentation with Intense Pulsed Light (IPL). Journal of Japan Society of Plastic and Reconstructive Surgery, 12:846 - 852.

10: Sugimoto I: Q-switched laser. In: (Okada M, ed.) Medical Photonics. 2011, Optronics, Tokyo. pp 36 38.

11: Watanabe S: Laser. In: (Miyachi Y, Matsunaga K, Furukawa F, and Utsugi R ed.) Aesthetic Dermatology. 2009, Nanzando, Tokyo. pp 214 224.

12: Park JM, Tsao H, and Tsao S (2008): Combined Use of Intense Pulsed Light and Q-Switched Ruby Laser for Complex Dyspigmentation Among Asian Patients. Lasers in Surgery and Medicine, 40:128 133.

13: Yamashita T, Negishi K, Hariya T, Kunizawa N, Ikuta K, Yanai M, and Wakamatsu S (2006): Intense Pulsed Light Therapy for Superficial Pigmented Lesions Evaluated by Reflectance-Mode Confocal Microscopy and Optical Coherence Tomography. Journal of Investigative Dermatology, 126:2281 - 2286

14: Ozawa T, Sirakawa M, Tateishi C, Sunohara M. Yamasaki M, and Harada T: Keiseigeka ryouikini okeru lezano oyoto kongono tenbou. In: (Okada M, ed.) Medical Photonics. 2011, Optronics, Tokyo. pp $29-35$.

15: Weiss RA, Weiss MA, and Beasley KL (2002): Rejuvenation of Photoaged Skin: 5 Years Results with Intense Pulsed Light of the Face, Neck, and Chest. Dermatologic Surgery, 28:1115 - 1119.

16: Bitter PH Jr, and Goldman MP (2000): Nonablative Skin Rejuvenation Using Intense Pulsed Light. Lasers in Surgery and Medicine, Supplement 12:16.

17: Negishi K, Wakamatsu S, Kushikata N, Tezuka Y, Kotani Y, and Shiba K (2002): Full-face photorejuvenation of photodamaged skin by intense pulsed light with integrated contact cooling: initial experiences in Asian patients. Lasers in Surgery and Medicine, 30:298 - 305.

18: Feng Y, Zhao J, and Gold MH (2008): Skin Rejuvenation in Asian Skin: The Analysis of Clinical Effects and Basic Mechanisms of Intense Pulsed Light. Journal of Drugs in Dermatology, 7:273 279 .

19: Negishi K: IPL ni yoru non-ablative skin rejuvenation. In: (Miyachi Y, Matsunaga K, Furukawa F, and Utsugi R ed.) Aesthetic Dermatology. 2009, Nanzando, Tokyo. pp 252 - 257.

20: Chee SN, Lowe P, and Lim A (2015): Laser skin resurfacing: A patient-centered classification based on downtime. Australasian Journal of Dermatology, 56:186 - 191.

21: Tanaka Y, Tsunemi Y, and Kawashima M (2016): Objective Assessment of Intensive Targeted Treatment for Solar Lentigines Using Intense Pulsed Light With Wavelengths Between 500 and $635 \mathrm{~nm}$. Lasers in Surgery and Medicine, 48:30 35.

22: Yamashita R, Matsuo Y, Kondo K, and Toyama T (2010): Laser Surgery for Aging Skin Problems. The Journal of Japan Society for Laser Surgery and Medicine, 31, 1:36 - 41.

23: Takeuchi K, Negishi K, Kushikata C, and Wakamatsu S (2008): Q-switched laser chiryoni okeru enshougo shikisochinchakuno hasseiritsuno kentou. Aesthetic Dermatology, 18:77 - 83.

24: Satoh Y: Japanese Skin Type (J S T) (1991). Proceedings of the Japanese Committee for Sunlight Protection, 2:62 - 70.

25: Yoshida M, Kawara S, and Kawada A (2011): Efficacy of IPL (Intense Pulsed Light) Treatment for Senile Lentigines on the Dorsal Hand. The Journal of Japanese Society for Dermatologic Surgery, 20, 2:125 - 128 .

26: Imayama S: Hifu no roka. In: (Miyachi Y, Matsunaga K, Furukawa F, and Utsugi R ed.) Aesthetic Dermatology. 2009, Nanzando, Tokyo. pp $52-68$.

27: Kawada A, Asai M, Kameyama H, Sangen Y, Aragane Y, Tezuka T, and Iwakiri K (2002): Videomicroscopic and histopathological investigation of intense pulsed light therapy for solar lentigines. Journal of Dermatological Science, 29:91 - 96.

28: Negishi K: IPL chirou watashino kufu. In: (Iijima M, Shiohara T, and Terui T ed.) Monthy Book Derma. 2013, Zennihonbyouinshuppankai, Tokyo. pp $119-127$. 